\title{
Association of the Ribosomal Subunits as Studied by Time-Resolved Cryo-EM
}

\author{
Tanvir R. Shaikh", Aymen Yassin", Zonghuan Lu**, David Barnard", Xing Meng", Hisham \\ Mohamed", Toh-Ming Lu ${ }^{* *}$, Terry Wagenknecht", and Rajendra K. Agrawal \\ * Wadsworth Center, New York State Department of Health, Albany, NY 12201 \\ ** Center for Integrated Electronics, Rensselaer Polytechnic Institute, Troy, NY 12180
}

We have previously demonstrated fabrication of a microfluidic device that mixes two reactants and sprays the mixture onto an EM grid, which is then plunged into the cryogen (Lu et al., 2009). The minimum reaction time from the initial point of complete mixing to fixation in cryogen is estimated to be $9.4 \mathrm{~ms}$. The reaction that we are studying is the association of the E. coli $30 \mathrm{~S}$ and $50 \mathrm{~S}$ ribosomal subunits in the presence of $10-12 \mathrm{mM} \mathrm{Mg}^{2+}$. We show that passage through the mixer and sprayer does not impair translation activity (Fig. 1). The 3D reconstruction from a dataset of the 70S-like images (Fig. 2) shows missing or weak densities for the inter-subunit bridges B2c, B4, and B6 (according to Yusupov et al., 2001 nomenclature). However, central bridges B2a, B2b, $\mathrm{B} 3$, and B7a were present in this reconstruction. Since all these bridges are intact in the map of control, pre-associated $70 \mathrm{~S}$ ribosomes that were also passed through the same microfluidic device, our results show that we are able to capture possible intermediates of association. Furthermore, subclasses derived after the application of the three-dimensional maximum likelihood classification method (ML3D) show variability in peripheral bridges B1a, B7b, and "X" (the latter according to Gabashvili et al., 2000 nomenclature), such that the presence of two of these three bridges was anticorrelated with the presence of the third. These results suggest that during association, the central bridges form first, while the small subunit teeters atop the large subunit, and then finally both ribosomal subunits adopt their final conformation within the fully associated 70S ribosome.

We are currently processing data from a device which allows a significantly longer reaction time, $42 \mathrm{~ms}$. By using cryo-EM data from mixing devices with varying delay times, we hope to formulate a more complete model of the sequence of events during subunit association. In addition, we are testing modifications in the preparation of sprayed grids that could improve the yield of usable image data.

Also, we are attempting to improve the yield of image data. A limiting factor with the yield is the presence of droplets which are spread thinly enough to image. For the data set from the $9 \mathrm{~ms}$ device, we exhaustively searched 19 grids to collect 303 micrographs, to obtain about 17,000 particle images, of which $\sim 26 \%$ were classified as 70 S-like. Furthermore, about 60-fold higher concentration is necessary than in conventional blotting techniques, and about 5-fold more volume is needed per grid. We will describe modifications to our grid-preparation methodologies to improve the yield of imaging of sprayed specimens.

\section{References.}

1. Gabashvili I.S., Agrawal R.K., Spahn C.M., Grassucci R.A., Svergun D.I., Frank J., Penczek P. (2000) Solution structure of the E. coli $70 \mathrm{~S}$ ribosome at $11.5 \AA$ resolution. Cell. 100: 537-49.

2. $\quad$ Lu Z., Shaikh T.R., Barnard D., Meng X., Mohamed H., Yassin A., Mannella C.A., Agrawal R.K., Lu T.-M., and Wagenknecht T. "(2009) Monolithic Microfluidic Mixing-Spraying Devices for Time-Resolved CryoElectron Microscopy.” J Struct Biol. 168: 388-95.

3. Yusupov M.M., Yusupova G.Z., Baucom A., Lieberman K., Earnest T.N., Cate J.H., Noller H.F. (2001) Crystal structure of the ribosome at 5.5 A resolution. Science. 292: 883-9 


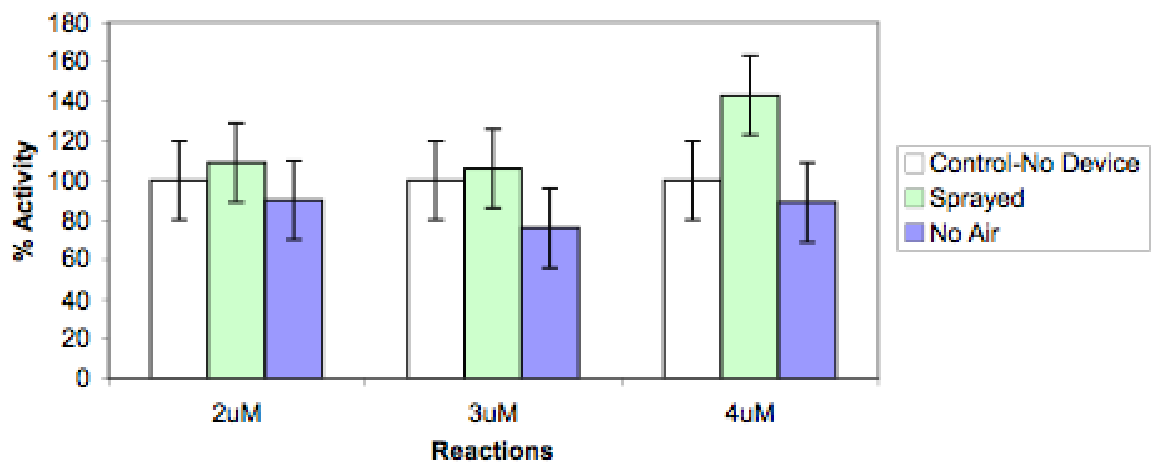

Figure 1. Cell-free translation activity of sprayed 70S. Activity of the ribosomes at different concentrations was determined by collecting the spray and assaying using a cell free translation system (PURESYSTEM $\Delta 1$ Ribosomes, Cosmo Bio Co. Ltd, Japan). Comparison is made against $30 \mathrm{~S}$ and $50 \mathrm{~S}$ subunits associated to $70 \mathrm{~S}$ ribosomes on a sucrose gradient (regarded as the control). The results showed similar activity compared to $70 \mathrm{~S}$ ribosomes associated from a gradient.

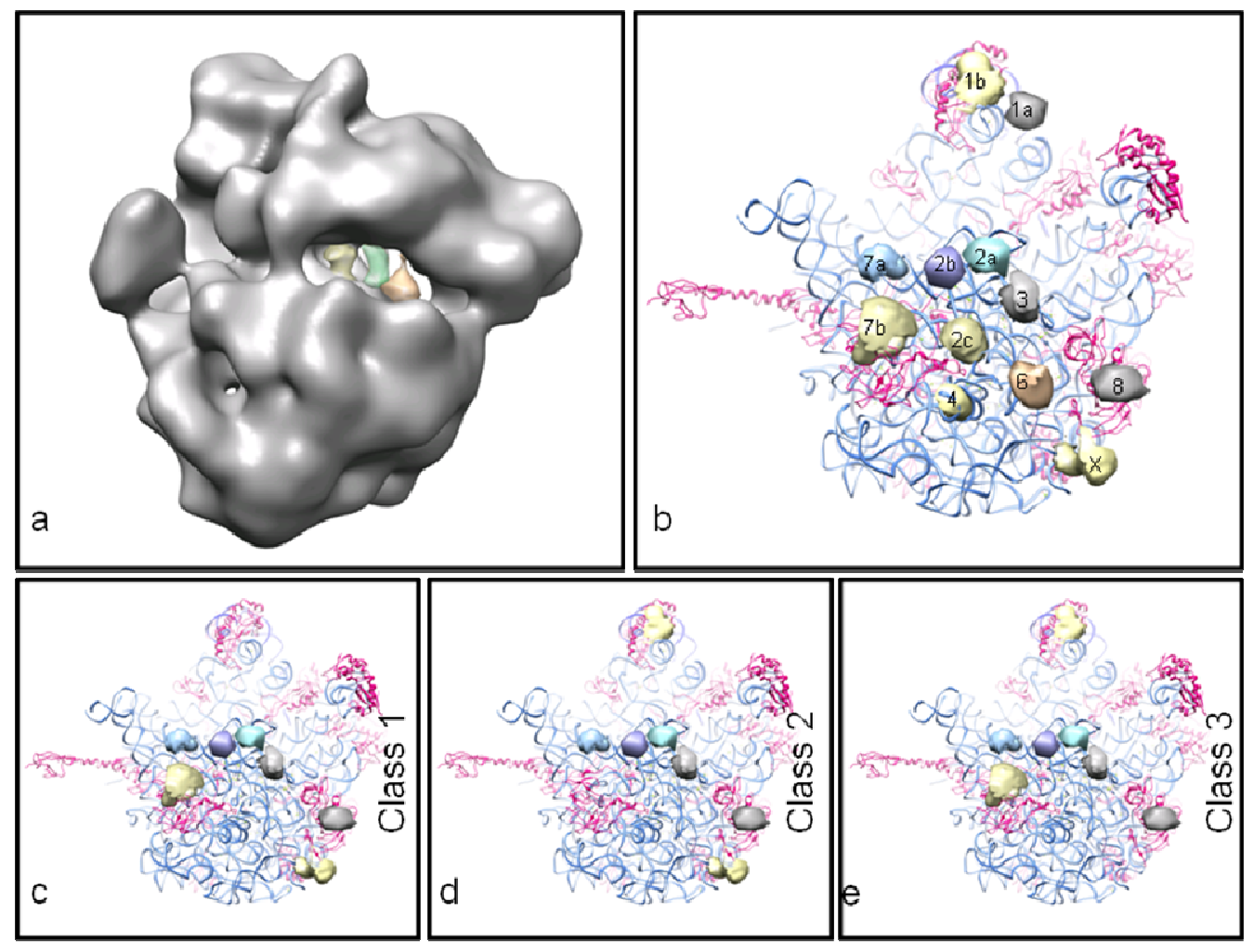

Figure 2. Bridge formation at 9 milliseconds. (a) Three bridges missing from a reconstruction of 4,445 particle images - B2c (yellow), B4 (green), and B6 (orange) - have been segmented from a pre-associated control 70S reconstruction. (b) View along intersubunit space of the large subunit, with bridges labeled. (c-e) Summary of variability of bridges following classification by ML3D. Of bridges $\mathrm{B} 1 \mathrm{~b}, \mathrm{~B} 7 \mathrm{~b}$, and $\mathrm{BX}$, the presence of two of these bridges precludes the presence of the third. 